\title{
Semiquantal molecular dynamics simulations of hydrogen-bond dynamics in liquid water using spherical gaussian wave packets
}

\section{AUTHOR(S):}

Ono, Junichi; Kim, Hyeon-Deuk; Ando, Koji

\section{CITATION:}

Ono, Junichi ... [et al]. Semiquantal molecular dynamics simulations of hydrogen-bond dynamics in liquid water using spherical gaussian wave packets. International Journal of Quantum Chemistry 2013, 113(3): 356-365

\section{ISSUE DATE:}

2013-02-05

URL:

http://hdl.handle.net/2433/217065

\section{RIGHT:}

This is the accepted version of the following article: [Ono, J., Hyeon-Deuk, K. and Ando, K. (2013), Semiquantal molecular dynamics simulations of hydrogen-bond dynamics in liquid water using spherical gaussian wave packets. Int. J. Quantum Chem., 113: 356-365. ], which has been published in final form at http://dx.doi.org/10.1002/qua.24146. This article may be used for non-commercial purposes in accordance with Wiley Terms and Conditions for Self-Archiving.; This is not the published version. Please cite only the published version: この論文は出版社版でありません。引用の際 には出版社版をご確認ざ利用ください。 


\title{
Semiquantal Molecular Dynamics Simulations of Hydrogen-Bond Dynamics in Liquid Water using Spherical Gaussian Wave Packets*
}

\author{
Junichi Ono, Kim Hyeon-Deuk, and Koji Ando \\ Department of Chemistry, Kyoto University, Kyoto 606-8502, Japan
}

\begin{abstract}
A semiquantal (SQ) molecular dynamics (MD) simulation method using spherical Gaussian wave packets (WPs) is applied to a microscopic analysis of hydrogen-bond (H-bond) exchange dynamics in liquid water. We focus on the molecular jump mechanism of H-bond reorientation dynamics proposed from a classical MD simulation by Laage and Hynes (Science 2006, 311, 832). As a notable quantum effect, broadenings of both the oxygen and hydrogen WPs of jumping water are observed associated with the H-bond switching events. Nonetheless, quantum effects on averaged trajectories of structural parameters measured with respect to the WP centers are rather minor. A $1 / f$ fluctuation of local H-bond number is observed in both SQ and classical simulations. This is obtained straightforwardly from the real-time trajectories, in contrast with the originally found $1 / f$ fluctuation (Sasai et al., J. Chem. Phys. 1992, 96, 3045) of the total potential energies collected at quenched inherent structures. The quantum effects are found to accelerate the relaxation of H-bond number fluctuation, which is reflected in the region near the lower bound of the $1 / f$ behavior in the power spectra. New developments in the implementation of SQMD simulations including all atoms are also described.
\end{abstract}

\section{Introduction}

Elucidating hydrogen-bond (H-bond) network dynamics in water and its relevance to chemical and biological processes remains to be an open problem ${ }^{1-6}$. The unique topology of aqueous H-bond network suggests an existence of collective order with frustrated partial disorder in liquid state ${ }^{7}$. The hydrophobic interaction is another peculiarity of the solvent in which the H-bond network plays a determining role. While the molecular dipole moment of an isolated water monomer is smaller than 2 Debye, the liquid dielectric response is larger (and also faster ${ }^{8}$ ) than in other solvents of comparable molecular dipole. These are expected to offer keys to understanding of structures, fluctuations, and functions of proteins and other biological molecules, but their molecular pictures are yet unclear.

Since the major component of H-bond dynamics consists of motions of light hydrogen atoms, quantum effects should not be neglected ${ }^{9-13}$. Nonetheless, classical mechanical molecular dynamics (MD) simulations have been actively employed to provide intriguing pictures. The use of classical MD may be justified qualitatively by the anticipated decoherence of quantum effects in condensed phase at temperatures as high as the ambient one. The cooperative nature of $\mathrm{H}$-bond network dynamics may also render an effective harmonic picture of collective $\operatorname{modes}^{8}$, which would then support the significance of classical dynamics.

Nevertheless, inclusion of quantum effects into condensed phase simulations has been a subject of active research. An indirect and useful way is to convert classical timecorrelation functions (TCFs) to quantum ones by assuming some quantum-classical correspondences between their Fourier transforms ${ }^{14-16}$. More recently, a class of methods based on the imaginary-time path-integral theory is at-

*International Journal of Quantum Chemistry 2013, 113, 356-365 DOI: 10.1002/qua.24146 taining increasing popularity ${ }^{17-20}$. They are computationally tractable and have shown to give reliable TCFs under certain conditions $^{21,22}$. This last feature is attractive since TCFs are directly related to observed quantities via the linear response theory ${ }^{23}$.

With respect to the real-time and real-space dynamics and inclusion of quantum phase, which are rather obscure in the imaginary-time approaches, the semiclassical wave packet (WP) methods, in particular, those with the coherentstate basis and the initial value representation (IVR) of propagators $^{24,25}$ appear promising. Their current bottleneck due to high computational cost may be resolved with proper approximations, e.g., some pre-averaging by exploiting the decoherence in condensed phases. Other promising approaches include the family of multi-configuration timedependent Hartree (MCTDH) method ${ }^{26}$ which can now deal with hundreds of degrees of freedom ${ }^{27}$.

In this work, we apply a recently developed semiquantal (SQ) MD simulation method ${ }^{28,29}$ to real-time and real-space microscopic analysis of H-bond dynamics in water. The theoretical basis is similar to the thawed Gaussian WP method ${ }^{30}$, but is distinguished by the canonical Hamiltonian form of the equations of motion for both the WP center and width variables. Its conceptual and practical merits have been illustrated by analytical theories ${ }^{31-33}$ and MD simulations ${ }^{28,29}$. The canonical Hamiltonian form is also shared with the expectation value approaches of moments $\mathrm{s}^{34}$ and cumulants ${ }^{35}$. Although the WP approaches will avail more straightforward inclusion of quantum phase, we reserve it for future investigations. Nevertheless, the zero-point and shallow-tunneling effects are taken into account via the dynamic WP broadening.

In the analysis of H-bond dynamics, we follow the work by Laage and Hynes ${ }^{36}$ which shed light on a molecular jump mechanism of water reorientation dynamics by a classical MD simulation. Recently, experimental support for the molecular jump mechanism in aqueous solution has also been provided using multi-dimensional vibrational spectroscopy ${ }^{37}$. Within the framework of the present approximation, the quantum ef- 
fects on the H-bond exchange dynamics are assessed. In addition, a $1 / f$ fluctuation of local H-bond number is discussed in relation to the previously found $1 / f$ behavior of total potential energies sampled at quenched inherent structures ${ }^{38}$.

The next section summarizes the computational method. The results and discussion are then presented. The final section concludes.

\section{Computation}

\section{Semiquantal MD Simulation}

The SQMD simulation method has been described previously ${ }^{28,29}$, in which only hydrogen atoms were quantized using spherically symmetric Gaussian WPs as a first approximation. Although it has been generally considered that the quantization of hydrogen atoms plays a dominant role in liquid water due to the light mass, the quantum role of oxygen atoms has remained unclear. The path-integral Monte Carlo simulations using the rigid water model have shown that the effect of center-of-mass quantization, which nearly corresponds to the quantization of oxygen atoms, on the structural and energetic properties of liquid water is small but non-negligible ${ }^{39}$. Moreover, the recent experimental results of the static structure factors of liquid water from high energy x-ray diffraction have shown that the magnitude of the structural isotope difference between $\mathrm{H}_{2}{ }^{16} \mathrm{O}$ and $\mathrm{H}_{2}{ }^{18} \mathrm{O}$ is approximately one quarter of that between $\mathrm{H}_{2} \mathrm{O}$ and $\mathrm{D}_{2} \mathrm{O}$ at $26{ }^{\circ} \mathrm{C}^{40}$. In the present work, therefore, we examine the importance of quantizing oxygen atoms by comparing different levels of quantization: the fully-classical water (FCW), only $\mathrm{H}$ atoms quantized water (H-SQW), and both $\mathrm{O}$ and $\mathrm{H}$ atoms quantized water $(\mathrm{OH}-$ SQW) models.

For the potential force-field, we employ the flexible and non-polarizable q-SPC/Fw model ${ }^{41}$ that has been designed for the centroid MD (CMD) simulations to remove the doublecounting of quantum effects in the conventional empirical models for classical simulations. To compute the SQ potential, the intramolecular bending function is expanded to the second order, whereas the intramolecular stretching and intermolecular Coulomb potentials are computed to the full order. The Lennard-Jones (LJ) function between the oxygen sites, quantized only for the OH-SQW model, is expanded to the fourth-order. Their specific forms are presented in Appendix.

The simulations are carried out for 256 water molecules contained in a cubic box of length $19.7 \AA$ at the density of
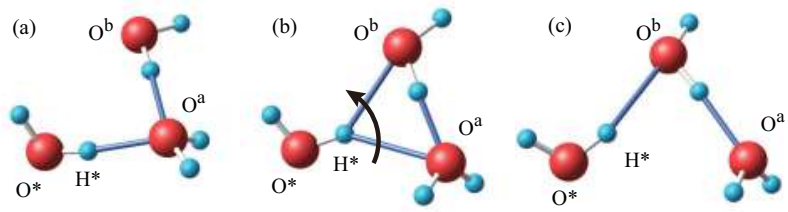

FIG. 1: Schematic drawing of the hydrogen-bond (H-bond) exchange event. (a) initial, (b) intermediate, and (c) final $\mathrm{H}$-bond state.

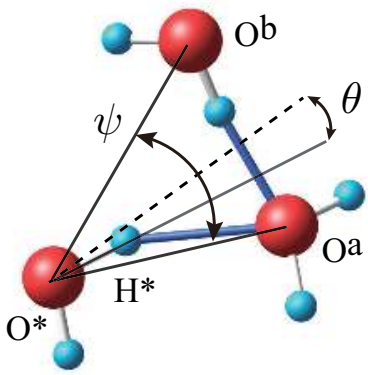

FIG. 2: Definition of structural parameters: the jump angle $\theta$ and the $\mathrm{O}^{a} \mathrm{O}^{*} \mathrm{O}^{b}$ angle $\psi$.

$0.997 \mathrm{~g} / \mathrm{cm}^{3}$ with the periodic boundary condition. The LJ interactions were smoothly truncated at the cut-off distance of half the box length with the smooth cut-off function, whereas the Ewald summation was employed for the Coulomb interactions.

The equations of motion are integrated with the velocity Verlet method with the time step of $0.1 \mathrm{fs}$. The system is equilibrated to the kinetic temperature of $298 \mathrm{~K}$ by the velocity scaling of the classical degrees of freedom. Then, microcanonical simulations were carried out for $2 \mathrm{~ns}$, from which the results were derived. The total energy was conserved within the standard deviation of $0.02 \mathrm{kcal} / \mathrm{mol}$, and the average kinetic temperature was $298 \mathrm{~K}$ with the standard deviation of $7 \mathrm{~K}$ for all the FCW, H-SQW, and $\mathrm{OH}-\mathrm{SQW}$ simulations.

\section{H-bond Exchange Events}

The H-bond switching event is illustrated in Fig. 1. We exclusively focus on this particular process and leave other possible pathways out of the scope of this study. We follow Ref. ${ }^{36}$ for the definition of structural parameters: the jump angle $\theta$ is the angle between the projection of the $\mathrm{O}^{*} \mathrm{H}^{*}$ vector on the $\mathrm{O}^{a} \mathrm{O}^{*} \mathrm{O}^{b}$ plane and the bisector of the $\mathrm{O}^{a} \mathrm{O}^{*} \mathrm{O}^{b}$ angle, $\phi$ is the out-of-plane angle between the $\mathrm{O}^{*} \mathrm{H}^{*}$ bond and the $\mathrm{O}^{a} \mathrm{O}^{*} \mathrm{O}^{b}$ plane, and $\psi$ is the $\mathrm{O}^{a} \mathrm{O}^{*} \mathrm{O}^{b}$ angle (see Fig. 2). In the SQMD simulations, the structural parameters were calculated with respect to the WP centers.

The number of H-bonds is counted according to the geometric definition $^{42}$ : the $\mathrm{O}-\mathrm{O}$ distance smaller than $3.5 \AA$, the $\mathrm{O}-\mathrm{H}$ distance smaller than $2.45 \AA$, and the $\mathrm{H}-\mathrm{O}-\mathrm{O}$ angle smaller than $30 \mathrm{deg}$.

\section{Results and Discussion}

\section{Static Equilibrium Properties}

We first calculate average monomer properties in liquid water: the $\mathrm{O}-\mathrm{H}$ bond length $r_{\mathrm{OH}}$, the $\mathrm{H}-\mathrm{O}-\mathrm{H}$ bond angle $\theta_{\mathrm{HOH}}$, and the molecular dipole moment $\mu$. A comparison between the present SQ and classical results and the previous one from 
TABLE I: Static equilibrium monomer properties in liquid water obtained from SQ and classical simulations using the $\mathrm{q}-\mathrm{SPC} / \mathrm{Fw}$ model. The number in parentheses represents the standard errors in the last digits.

\begin{tabular}{lccc}
\hline \hline & $r_{\mathrm{OH}}(\AA)$ & $\theta_{\mathrm{HOH}}(\mathrm{deg})$ & $\mu(\mathrm{D})$ \\
\hline OH-SQW & $1.0096(1)$ & $106.43(1)$ & $2.4375(1)$ \\
H-SQW & $1.0116(1)$ & $106.33(1)$ & $2.4452(1)$ \\
FCW & $1.0201(1)$ & $106.12(1)$ & $2.4716(1)$ \\
\hline PIMD $^{\mathrm{a}}$ & $1.019(1)$ & $106.2(1)$ & $2.465(1)$ \\
\hline \hline \multicolumn{4}{c}{${ }^{\mathrm{a}}$ Reference $^{43}}$.
\end{tabular}

the imaginary-time path-integral molecular dynamics (PIMD) simulations $^{43}$ is given in Table I. The average monomer properties in the SQMD simulations were calculated with respect to the WP centers. It is seen that the differences between the SQ and classical models are small; the bond length systematically decreases and the bond angle increases as the model becomes more quantal. These geometric changes can be qualitatively understood from the intramolecular extended SQ potential. Indeed, the H-SQW and OH-SQW models of an isolated water monomer yielded the energy minimum at shorter $\mathrm{O}-\mathrm{H}$ distances by 0.0075 and $0.0089 \AA$ and larger $\mathrm{H}-\mathrm{O}-\mathrm{H}$ angles by 0.19 and $0.24^{\circ}$, respectively, than the classical model. This is mainly due to the cross term between the variables of the WP centers and the WP width in Eqs. (A25) and (A27) in Appendix. As a result of these geometric changes, the average monomer dipole moments in the H-SQW and OH-SQW models become smaller than the classical one by approximately 1.1 and $1.4 \%$, respectively. As will be seen below, the smaller dipole moments in the SQ models lead to weaker intermolecular interactions which disorder the $\mathrm{H}$-bond structure and increase the translational mobility.

We next calculate the atom-atom radial distribution functions (RDFs) to investigate the relative quantum contributions of oxygen and hydrogen atoms to the local H-bond structure. In contrast to our previous studies ${ }^{28,29}$, the WP spreading effects are explicitly included in the present calculations of the quantum RDFs (see Appendix). The results are shown in Fig. 3. Specific differences in the oxygen-oxygen $(\mathrm{O}-$ $\mathrm{O})$, oxygen-hydrogen $(\mathrm{O}-\mathrm{H})$, and hydrogen-hydrogen $(\mathrm{H}-\mathrm{H})$ RDFs are seen between the quantum and classical models; in all cases, the peak heights systematically decrease as the model becomes more quantum mechanical. Comparing $\mathrm{H}-$ SQW with FCW in Fig. 3b, for example, the intramolecular peak of the O-H RDF becomes noticeably broader due to the WP delocalization of hydrogen atoms. In addition, the first intermolecular peak of the O-H RDF exhibits a considerable reduction of the intensity and a shift of the position toward the larger distance when hydrogen atoms are quantized: from 1.74 located at $1.72 \AA$ for FCW to 1.36 at $1.76 \AA$ for H-SQW. If we calculate the quantum O-H RDF based only on the WP centers in the H-SQW model ${ }^{28}$, the intensity of the first intermolecular peak becomes 1.63 at $1.73 \AA$ (see Fig. 4b). Therefore, the reduction of the peak intensity is mainly attributed to the WP spreading effects of hydrogen atoms on the observable distribution function, and the quantization of hydrogen atoms slightly disorders the WP centers in the local H-bond struc-
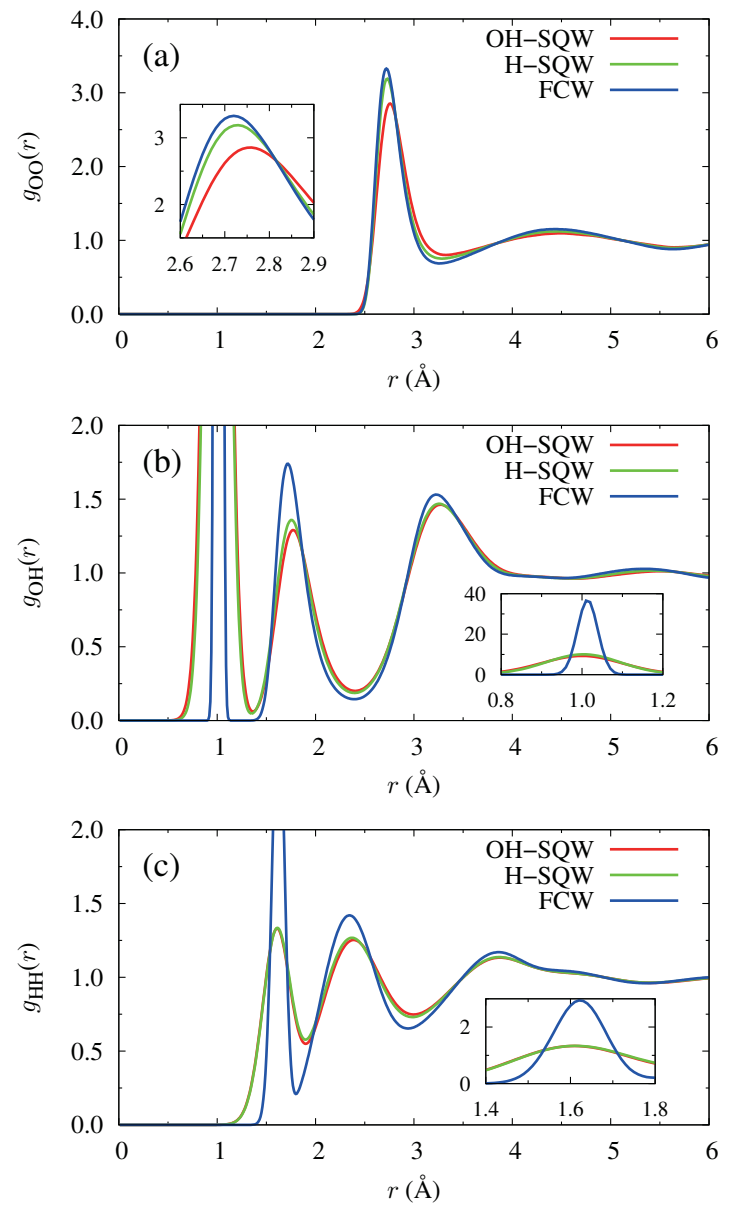

FIG. 3: Radial distribution functions for (a) O-O, (b) $\mathrm{O}-\mathrm{H}$, and (c) $\mathrm{H}-\mathrm{H}$ atom pairs. The insets show the magnifications of the corresponding first peaks.

ture. Note that the analogous quantum effects of hydrogen atoms can be observed in the H-H RDFs (see Figs. 3c and 4c). On the other hand, the difference between the two quantum models is significant in the O-O RDFs rather than in the O-H and H-H RDFs. In particular, the height of the first peak of the O-O RDF significantly decreases due to the quantization of oxygen atoms: from 3.19 located at $2.73 \AA$ for H-SQW to 2.85 at $2.76 \AA$ for $\mathrm{OH}-\mathrm{SQW}$. If we neglect the WP spreading effects in the calculations of the quantum O-O RDF, the corresponding peak height becomes 3.11 at $2.74 \AA$ for $\mathrm{OH}-\mathrm{SQW}$ (see Fig. 4a). Therefore, the decrease of the peak height is mainly attributed to the WP delocalization of oxygen atoms, and the quantization of oxygen atoms gives rise to the slight structural difference in the WP centers.

Overall, our results by the OH-SQW model are in good agreement with the previous PIMD simulations (see Fig. 5 in Ref. ${ }^{41}$ and Figs. 1-3 in Ref. ${ }^{43}$ ), except that the present intramolecular peak heights of the quantum O-H and H-H RDFs are slightly smaller. The small discrepancies in the RDFs and the average monomer properties in Table I may stem from the spherical Gaussian WP approximations in the present SQMD 

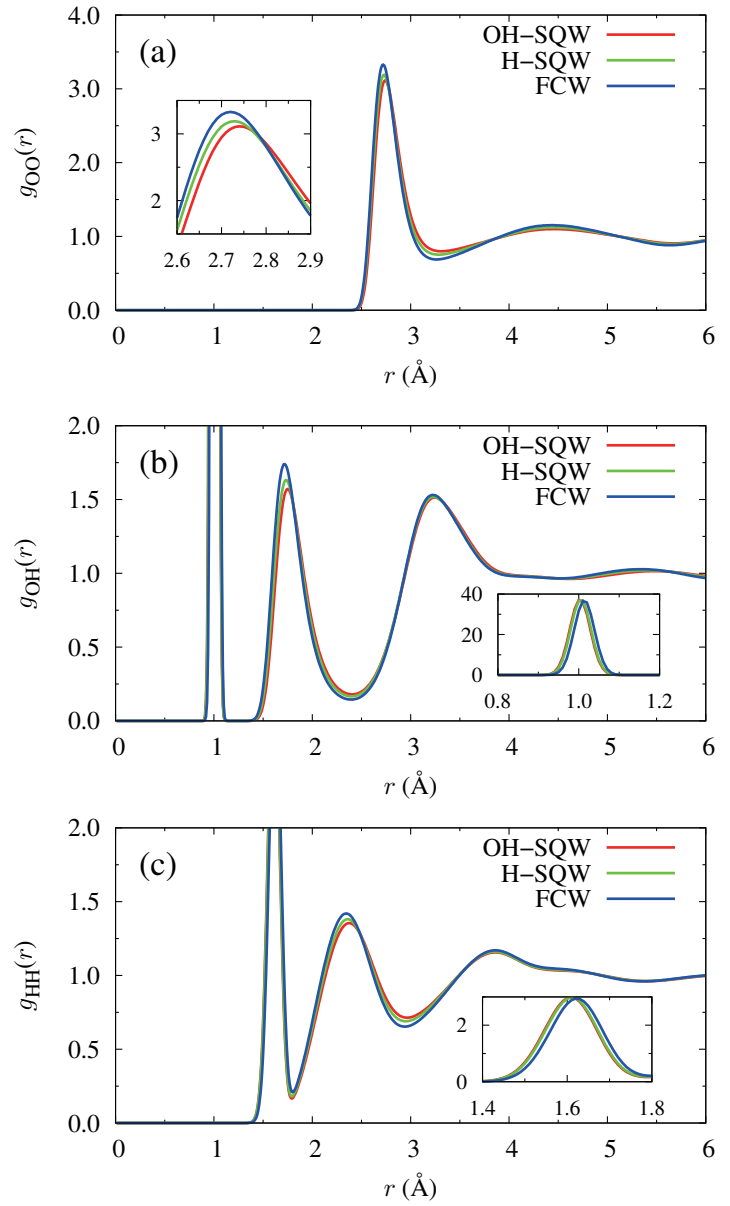

FIG. 4: Radial distribution functions calculated from the WP centers for (a) $\mathrm{O}-\mathrm{O}$, (b) $\mathrm{O}-\mathrm{H}$, and (c) $\mathrm{H}-\mathrm{H}$ atom pairs obtained from SQMD simulations. Also shown are the classical results in Fig. 3. The insets show the magnifications of the corresponding first peaks.

simulations, and could then be remedied by employing more general Gaussian WPs.

\section{Self-diffusion Coefficient}

We next compute the self-diffusion coefficient $D$ from the slope of the mean-square displacement

$$
D=\lim _{t \rightarrow \infty}\left\langle|\boldsymbol{r}(t)-\boldsymbol{r}(0)|^{2}\right\rangle_{0} / 6 t
$$

where $\langle\cdots\rangle_{0}$ denotes the statistical average and $\boldsymbol{r}(t)$ represents the positions of center-of-mass of molecule at time $t$. The results are listed in Table II. The quantum acceleration from the FCW is about $30 \%$ by the H-SQW and $48 \%$ by the OH-SQW, indicating the significance of quantization of oxygen atoms. The result of OH-SQW agrees well with the previous CMD simulations which accounted for both $\mathrm{O}$ and $\mathrm{H}$ quantum effects.
TABLE II: Computed self-diffusion coefficient in $\AA^{2} / p$ s from SQ and classical simulations with the $\mathrm{q}-\mathrm{SPC} / \mathrm{Fw}$ model. The standard errors in the final digits are given in parentheses.

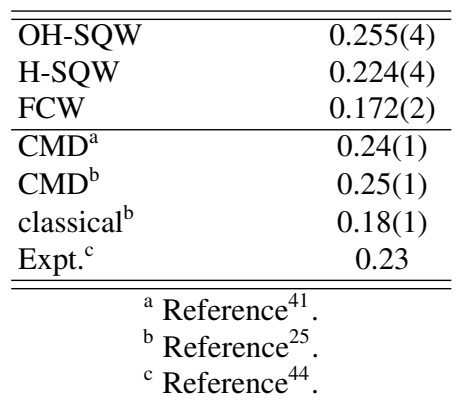

\section{H-bond Exchange Dynamics}

Here we examine the H-bond exchange trajectories. The points at which $\theta=0$ are taken to be the time origin $t=0$ of the H-bond exchange trajectories. From the microcanonical simulations of $2 \mathrm{~ns}$, ca 113,000, 131,000, and 143,000 $\mathrm{H}$-bond exchange events were sampled for FCW, H-SQW, and OH-SQW models, which means that the events involving three molecules are found in every 1.51, 1.30, and 1.19 ps, respectively. From the ratio of number of events, reduction of effective free energy barrier of $\mathrm{H}$-bond exchange due to quantization is estimated to be $0.09 \mathrm{kcal} / \mathrm{mol}$ for $\mathrm{H}-\mathrm{SQW}$ and $0.14 \mathrm{kcal} / \mathrm{mol}$ for $\mathrm{OH}-\mathrm{SQW}$.

Figure 5 shows averaged trajectories of the jump angle $\theta$ and the $\mathrm{O}^{a} \mathrm{O}^{*} \mathrm{O}^{b}$ angle $\psi$. The quantum effects on the trajectory of $\theta$ are comparatively minor; in the SQ models, both the magnitude and the standard deviation of $\theta$ in the initial and final H-bond states are slightly greater than the classical counterparts, whereas those in the intermediate state at $t \simeq 0$ are almost equal to the classical ones. On the other hand, the differences in the time-evolution of $\psi$ between the SQ and classical models are clearer than those in $\theta$; both the magnitude and the standard deviation of $\psi$ in the SQ models are greater than the classical counterparts not only in the initial and final $\mathrm{H}$-bond states but also in the intermediate state. Furthermore, we found that the quantum effects on the out-of-plane angle $\phi$ are almost identical with those on the angle $\psi$ (and thus not included in the figure).

The behavior of $\psi$ in Fig. 5 is related to those of the OO distances shown in Fig. 6. The trajectories for $\mathrm{O}^{*} \mathrm{O}^{a}$ and $\mathrm{O}^{*} \mathrm{O}^{b}$ are symmetric about $t=0$. In the region $t<0$, the quantum effect is almost negligible in the initially $\mathrm{H}$-bonded $\mathrm{O}^{*} \mathrm{O}^{a}$ distance, but is notable for the other two distances that involve the entering $\mathrm{O}^{b}$ atom. When the quantum effects are included, the $\mathrm{H}$-bond acceptor $\mathrm{O}^{b}$ initiates the $\mathrm{H}$-bond exchange dynamics by ca $0.2 \AA$ distant from the donor $\mathrm{O}^{a}$ compared to the FCW case.

Figure 7 shows the trajectories of H-bond accepting number by $\mathrm{O}^{*}$ and $\mathrm{O}^{a}$. The results for $\mathrm{O}^{b}$ are symmetric to the $\mathrm{O}^{a}$ ones about $t=0$. Overall, the H-bond accepting number by $\mathrm{O}$ atoms is smaller by ca. 0.05 in the quantum simulations. This is uniformly observed throughout the exchange event for 

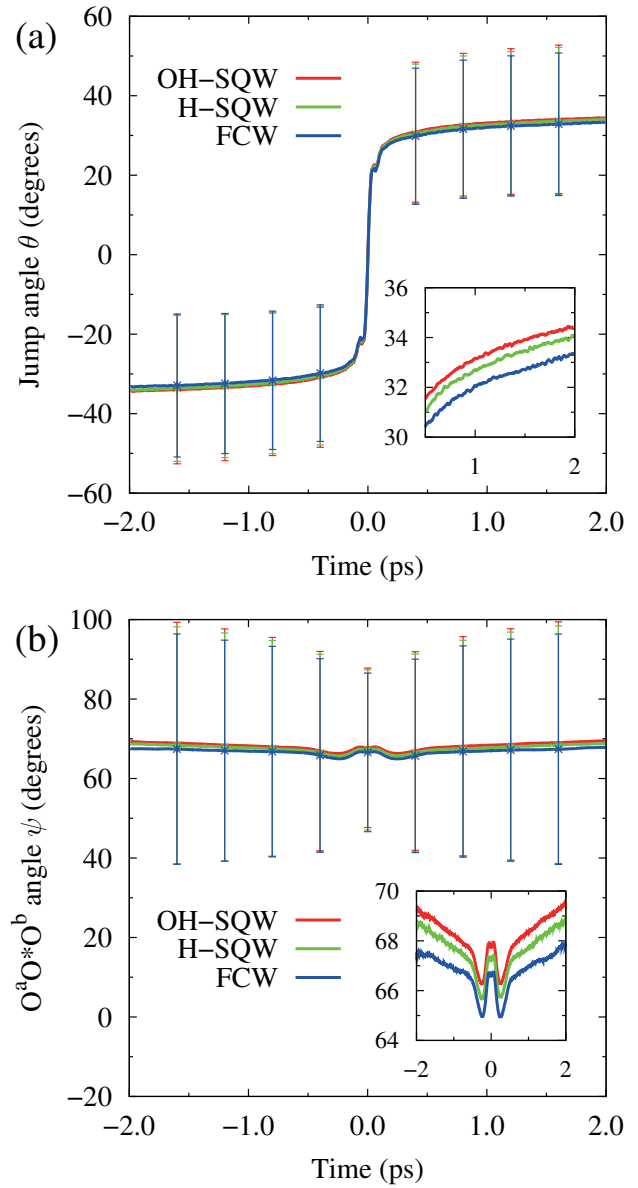

FIG. 5: Averaged trajectories of (a) the jump angle $\theta$ and (b) the $\mathrm{O}^{a} \mathrm{O}^{*} \mathrm{O}^{b}$ angle $\psi$. The standard deviations are also plotted as error bars.

$\mathrm{O}^{*}$ in Fig. 7a. Similar quantum effect is seen for the $\mathrm{O}^{a}$ case in Fig. $7 \mathrm{~b}$ in the time regions $t \leq 0$ and $t>0.5 \mathrm{ps}$, but between $t=0$ and 0.5 ps the quantum and classical trajectories overlap. This last behavior is symmetric to that of $\mathrm{O}^{b}$ in -0.5 ps $<t<0$. In this time region, $\mathrm{O}^{b}$ is releasing an old $\mathrm{H}$-bond to form a new one with $\mathrm{H}^{*}$. The results in Fig. $7 \mathrm{~b}$ show that this dynamics is nearly classical.

The trajectories of the WP widths are displayed in Fig. 8. The deviations from the statistical averages are plotted; for instance, the WP widths of $\mathrm{H}^{a}$ and $\mathrm{H}^{b}$ atoms in Fig. 8a around $t=-2 \mathrm{ps}$ and $2 \mathrm{ps}$ are close to the average value. The WP of $\mathrm{H}^{a}$ in the $\mathrm{H}$-bond releasing water slightly shrinks in $t<0$ toward $t=0$, sharply broadens at around $t=0$, and then gradually relaxes to the average value in $t>0$. This is symmetric about $t=0$ to the behavior of $\mathrm{H}^{b}$ in the $\mathrm{H}$-bond accepting water whose WP broadens toward $t=0$, sharply shrinks, and gradually broadens. The WP width of $\mathrm{H}^{*}$ atom is smaller than the average before the $\mathrm{H}$-bond exchange, but sharply broaden toward the intermediate state at $t=0$. The qualitative behaviors of the WP widths of oxygen atoms are similar to those of hydrogens, but before the H-bond exchange the WP widths of $\mathrm{O}^{*}$ and $\mathrm{O}^{a}$ are close to the average whereas that of $\mathrm{O}^{b}$ is
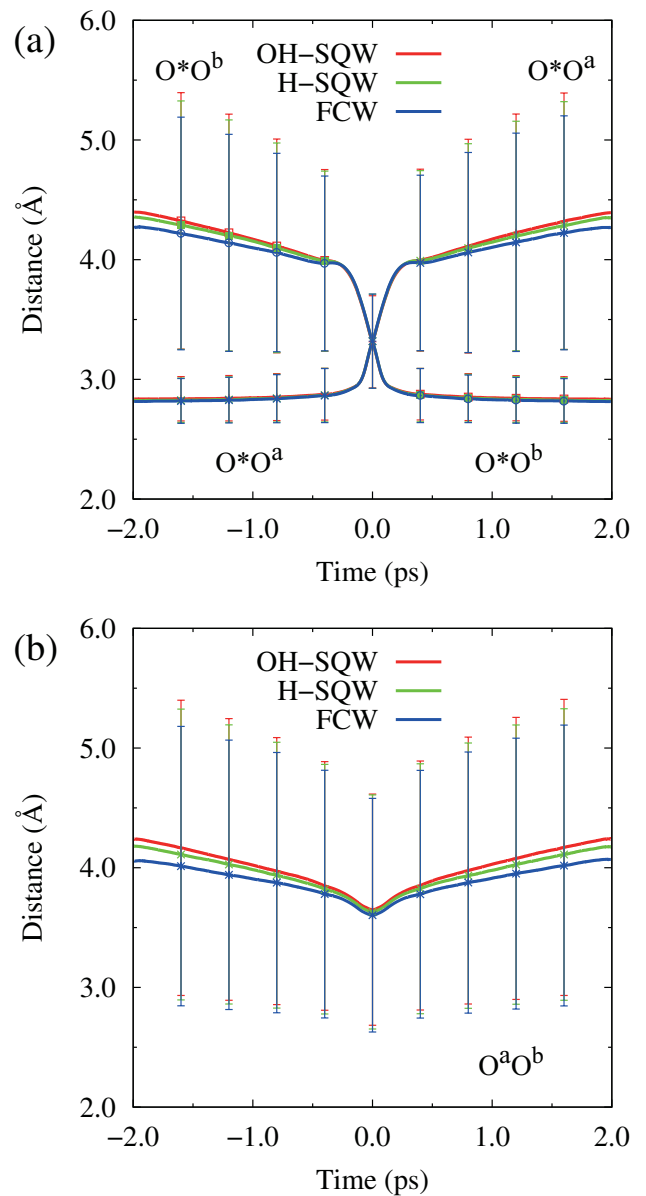

FIG. 6: Averaged trajectories of (a) $\mathrm{O}^{*} \mathrm{O}^{a}$ and $\mathrm{O}^{*} \mathrm{O}^{b}$, and (b) $\mathrm{O}^{a} \mathrm{O}^{b}$ distances. The standard deviations are also plotted as error bars.

broader. The behaviors of WP widths appear correlated with those of $\mathrm{H}$-bond coordination numbers shown in Fig. 7. The $\mathrm{H}$-bond accepting $\mathrm{O}^{b}$ has less $\mathrm{H}$-bonds and broader WP width than average prior to the $\mathrm{H}$-bond exchange.

\section{H-bond Number Fluctuation}

Finally, we analyze the dynamics of local H-bond number $N_{\mathrm{HB}}(t)$ with focus on the $1 / f^{\alpha}$ behavior. Figure 9 shows the normalized TCFs of the fluctuation,

$$
C_{\mathrm{HB}}(t)=\left\langle\delta N_{\mathrm{HB}}(t) \delta N_{\mathrm{HB}}(0)\right\rangle_{0} /\left\langle\delta N_{\mathrm{HB}}^{2}\right\rangle_{0},
$$

where $\delta N_{\mathrm{HB}}(t)=N_{\mathrm{HB}}(t)-\left\langle N_{\mathrm{HB}}\right\rangle_{0}$, and the power spectra

$$
S_{\mathrm{HB}}(\omega)=\left|\int_{0}^{T} d t \mathrm{e}^{-i \omega t} N_{\mathrm{HB}}(t)\right|^{2},
$$

in which $T$ is the simulation time length.

The semi-log plots of TCF in Fig. 9a indicate that the relaxation involves multiple time scales. They also show that the quantum effect accelerates the decay. In the shorter time 

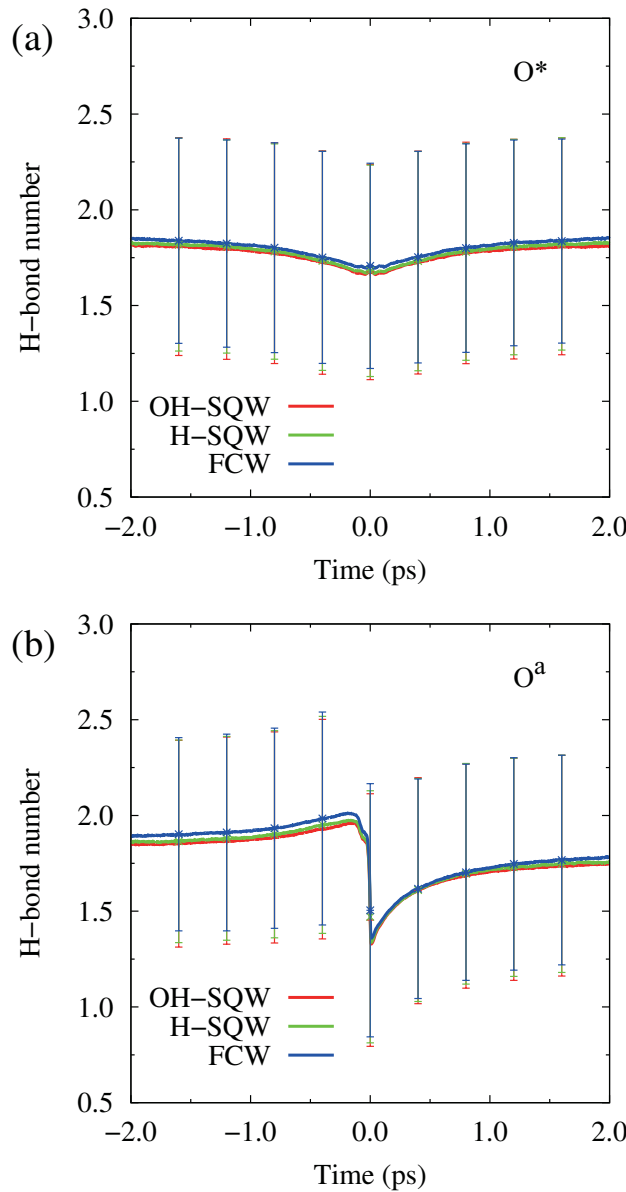

FIG. 7: Averaged trajectories of the H-bond accepting number of (a) $\mathrm{O}^{*}$ and (b) $\mathrm{O}^{a}$ atoms. The standard deviations are also plotted as error bars.

region than $\sim 0.1 \mathrm{ps}$, the decay profiles overlap well, whereas the difference becomes more evident in the longer time region where the slopes of the semi-log plots clearly deviate from each other. The difference in the longer time is reflected in the power spectra in Fig. 9b in which the different models deviate in the lower frequency region than $10 \mathrm{~cm}^{-1}$. In the higher frequency region up to ca $200 \mathrm{~cm}^{-1}$, the power spectra commonly follow the $1 / f$ behavior. After a plateau toward $\sim 400 \mathrm{~cm}^{-1}$, the spectra follow near the $1 / f^{2}$ behavior. As indicated by the dashed and dotted lines in Fig. $9 \mathrm{~b}$, the least-squares fit to $1 / f^{\alpha}$ of the region $20-1000 \mathrm{~cm}^{-1}$ yielded $\alpha=1.2$, whereas the fit of the region $1000-5000 \mathrm{~cm}^{-1}$ gave $\alpha=1.7$.

The power spectra in Fig. 9b exhibit the typical behavior of uniformly distributed relaxation times ${ }^{45}$, namely, a white noise region in low frequency, a $1 / f$ noise in the intermediate, and a $1 / f^{2}$ region in high frequency. If we assume this model and read from Fig. $9 \mathrm{~b}$ that the $1 / f$ behavior ranges between 20 $\mathrm{cm}^{-1}$ and $100 \mathrm{~cm}^{-1}$, the TCF of $N_{\mathrm{HB}}(t)$ is considered to be a uniform superposition of exponential decays with relaxation times ranging between $0.3 \mathrm{ps}$ and $1.7 \mathrm{ps}$. The plateau region of the spectra between $200 \mathrm{~cm}^{-1}$ and $400 \mathrm{~cm}^{-1}$ seems to come
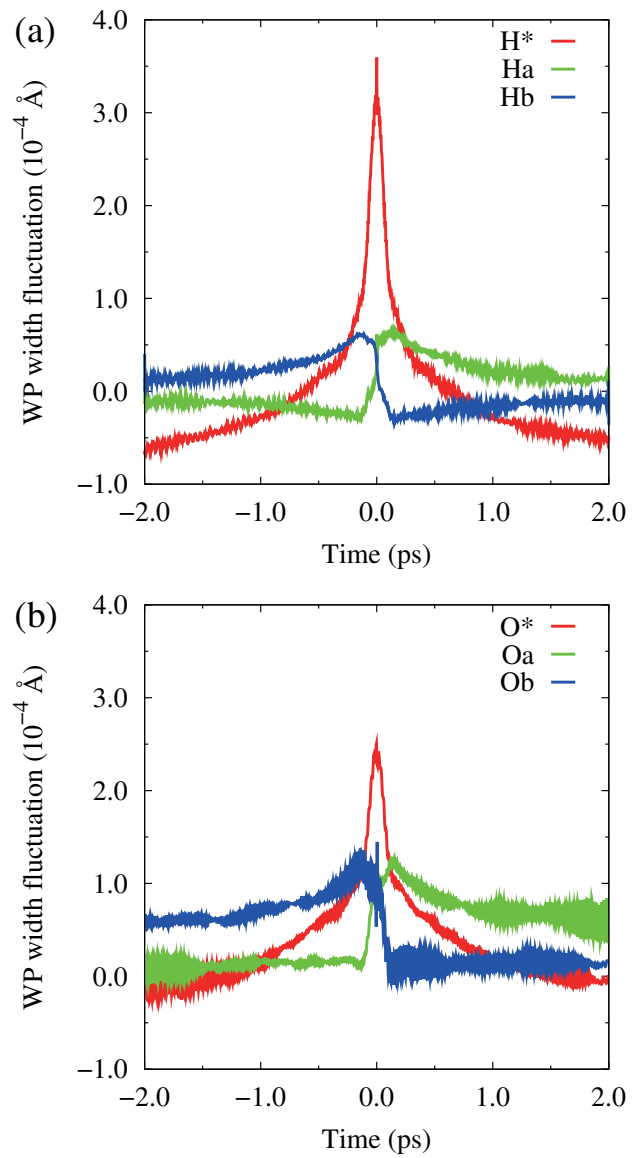

FIG. 8: Averaged trajectories of the wave packet (WP) width fluctuations of (a) $\mathrm{H}$ atoms and (b) $\mathrm{O}$ atoms in the OH-SQW model. The WP width fluctuation is defined by the difference from the statistical average.

from the small oscillation at $t \simeq 0.1 \mathrm{ps}$ in the TCFs of Fig. 9a, which would be assigned to the translational intermolecular stretching vibration of H-bonds. In Fig. 9b, traces of small peaks are seen around $1500 \mathrm{~cm}^{-1}$ and $3500 \mathrm{~cm}^{-1}$ which correspond to the intramolecular bending and stretching vibrations, although they are only indirectly related to the criteria of H-bond. The intermolecular librational motions (hindered rotations) are expected in the region between $400 \mathrm{~cm}^{-1}$ and $1000 \mathrm{~cm}^{-1}$, but the corresponding peaks are absent in Fig. $9 \mathrm{~b}$. This implies that the amplitudes of these motions are as small as to stay within the range of $\mathrm{H}$-bond criteria and thus are not much reflected in the $N_{\mathrm{HB}}(t)$ dynamics. Another interpretation would be that the librational motions are buried in a broad peak ranging from $200 \mathrm{~cm}^{-1}$ to $1000 \mathrm{~cm}^{-1}$. On the other hand, the larger amplitude $\mathrm{H}$-bond exchange motions have the time scales of $\sim 1 \mathrm{ps}$, in the range corresponding to the $1 / f$ fluctuation.

In a previous work by a classical MD simulation ${ }^{46}$, a crossover to the $1 / f^{2}$ behavior at $\sim 100 \mathrm{~cm}^{-1}$ was observed. This is dissimilar to our results particularly around the crossover region, which can stem from the different simulation conditions employed in Ref. ${ }^{46}$ such as the NVT ensem- 

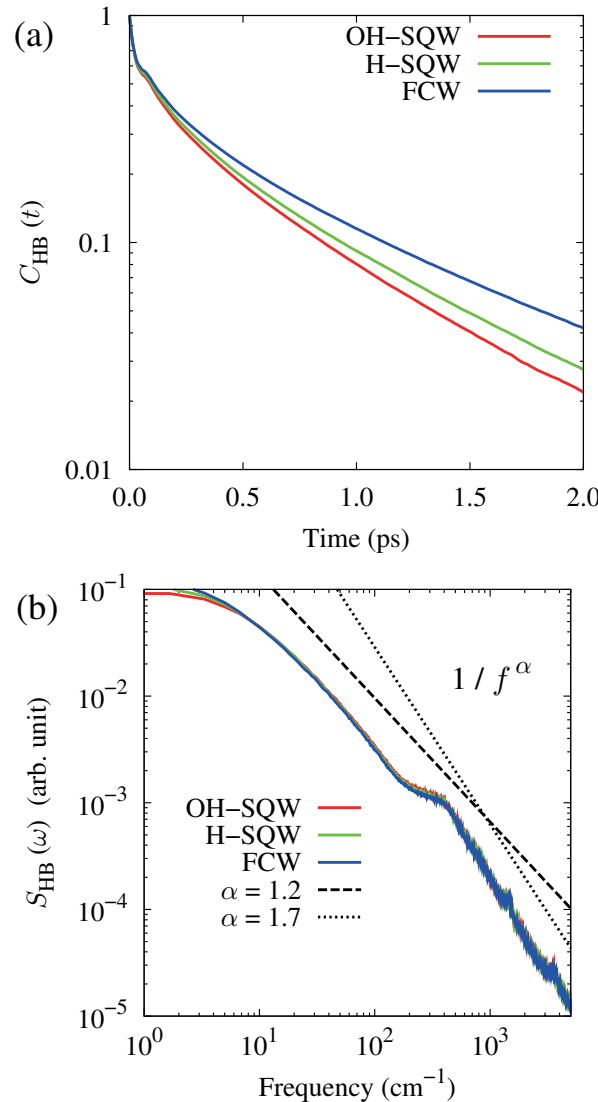

FIG. 9: (a) Time correlation functions and (b) power spectra of the local H-bond coordination number fluctuation. Note the log-scales of the axes.

ble, SPC/E force-field, and especially the SHAKE algorithm for intramolecular constraint and the definition of $\mathrm{H}$-bonds that depends only on the $\mathrm{O}-\mathrm{O}$ and $\mathrm{O}-\mathrm{H}$ distances ${ }^{47}$; the precise reason is however unclear at present.

\section{Concluding Remarks}

Quantum effects in H-bond exchange dynamics have been analyzed by the SQMD simulation. While the qualitative behaviors of structural parameters with respective to the WP centers, in particular that of the jump angle $\theta$, are found to be well captured by the classical simulation, salient dynamic broadenings of the WP widths associated with H-bond exchanges have been detected.

The quantum effects are also found to enhance the H-bond exchanges as measured from the relaxation profile of the local H-bond number fluctuations. This is reflected in the region near the lower bound of the $1 / f$ behavior in the power spectra. The origin of the $1 / f$ behavior and its crossover to $1 / f^{2}$ has been discussed. Nonetheless, to obtain more detailed microscopic picture, analysis of the potential energy surfaces along both the WP center and width coordinates will be needed, which is left open for future investigations.

\section{Acknowledgments}

J.O. is supported in part by the Global COE program "International Center for Integrated Research and Advanced Education in Material Science" (No. B-024). K.A. acknowledges supports from KAKENHI Nos. 20108017 (“ $\pi$-space”) and 22550012.

\section{Appendix: Computational Details}

\section{Semiquantal time-dependent Hartree theory}

Here we summarize the semiquantal time-dependent Hartree (SQTDH) theory for the SQMD simulation. In particular, Eq. (A12) generalizes the previous derivation ${ }^{28}$ for systematic and versatile implementations.

We assume the trial wave function of a Hartree product form

$$
\Psi\left(\mathbf{q}_{1}, \ldots, \mathbf{q}_{N}, t\right)=\prod_{j=1}^{N} \Psi_{j}\left(\mathbf{q}_{j}, t\right)
$$

where $\Psi_{j}$ is the basis function associated with the $j$ th quantum particle, and $\mathbf{q}_{j}$ denotes the position vector of the $j$ th particle in the three-dimensional Cartesian coordinate system. In the present study, we employ the spherical Gaussian form

$$
\begin{aligned}
& \Psi_{j}\left(\mathbf{q}_{j}, t\right)= \\
& N_{j}(t) \exp \left[A_{j}(t)\left|\mathbf{q}_{j}-\mathbf{r}_{j}(t)\right|^{2}+\frac{i}{\hbar} \mathbf{p}_{j}(t) \cdot\left(\mathbf{q}_{j}-\mathbf{r}_{j}(t)\right)\right],
\end{aligned}
$$

in which

$$
A_{j}(t)=-\frac{1}{4 \rho_{j}(t)^{2}}+\frac{i}{\hbar} \frac{\Pi_{j}(t)}{2 \rho_{j}(t)}
$$

and $N_{j}(t)=\left(2 \pi \rho_{j}(t)^{2}\right)^{-3 / 4}$ is the normalization factor. This trial function is specified by the time-dependent variational parameters $\left\{\mathbf{r}_{j}(t), \mathbf{p}_{j}(t), \rho_{j}(t), \Pi_{j}(t)\right\}$. The parameters $\mathbf{r}_{j}(t) \equiv\left(r_{j x}(t), r_{j y}(t), r_{j z}(t)\right)$ and $\rho_{j}(t)$ describe the center and the width of the $j$ th wave packet; $\mathbf{p}_{j}(t)$ and $\Pi_{j}(t)$ turn out to represent the conjugate momenta of $\mathbf{r}_{j}(t)$ and $\rho_{j}(t)$, respectively ${ }^{48}$. Their equations of motion are determined by the time-dependent variational principle, which result in the canonical Hamilton form with the extended Hamiltonian function,

$$
H_{\mathrm{ext}} \equiv\langle H\rangle=\sum_{j=1}^{N}\left[\frac{\left|\mathbf{p}_{j}\right|^{2}}{2 m_{j}}+\frac{3 \Pi_{j}^{2}}{2 m_{j}}+\frac{3 \hbar^{2}}{8 m_{j} \rho_{j}^{2}}\right]+\langle V\rangle
$$

in which $m_{j}$ is the mass of the $j$ th particle, and $\langle\ldots\rangle$ indicates the quantum mechanical expectation value defined by the wave function in Eq. (A1). 
The key task in the implementation is to evaluate the potential expectation $\langle V\rangle$. As in ordinary MD simulations, we assume the pairwise form

$$
V\left(\mathbf{q}_{1}, \ldots, \mathbf{q}_{N}\right)=\sum_{j>k}^{N} V_{j k}\left(\mathbf{q}_{j}, \mathbf{q}_{k}\right),
$$

where $V_{j k}\left(\mathbf{q}_{j}, \mathbf{q}_{k}\right) \equiv V_{j k}\left(\left|\mathbf{q}_{j}-\mathbf{q}_{k}\right|\right)$ denotes the pair potential function depending only on the interatomic distance. For some functional forms such as exponential and Gaussian functions, it is possible to perform Gaussian integrals analytically and obtain the exact potential expectation. Otherwise we expand $V_{j k}$ around $\left\langle\mathbf{q}_{j}\right\rangle=\mathbf{r}_{j}$ and $\left\langle\mathbf{q}_{k}\right\rangle=\mathbf{r}_{k}$ as

$$
\begin{aligned}
& V\left(\mathbf{q}_{1}, \ldots, \mathbf{q}_{N}\right)=\sum_{j>k}^{N} V_{j k}\left(\mathbf{r}_{j}+\Delta \mathbf{q}_{j}, \mathbf{r}_{k}+\Delta \mathbf{q}_{k}\right) \\
& =\left.\sum_{j>k}^{N} \sum_{n=0}^{\infty} \frac{1}{n !}\left(\sum_{l=j, k} \Delta \mathbf{q}_{l} \cdot \frac{\partial}{\partial \mathbf{r}_{l}^{\prime}}\right)^{n} V_{j k}\left(\mathbf{r}_{j}^{\prime}, \mathbf{r}_{k}^{\prime}\right)\right|_{\mathbf{r}^{\prime}=\mathbf{r}},
\end{aligned}
$$

where $\Delta \mathbf{q}_{l} \equiv \mathbf{q}_{l}-\left\langle\mathbf{q}_{l}\right\rangle$, and all the differentiations are performed at the corresponding WP centers. Using the following relations

$$
\begin{aligned}
\left\langle\left(\Delta q_{l x}\right)^{2 n}\right\rangle= & \left\langle\left(\Delta q_{l y}\right)^{2 n}\right\rangle \\
& =\left\langle\left(\Delta q_{l z}\right)^{2 n}\right\rangle=(2 n) ! \rho_{l}^{2 n} /\left(n ! 2^{n}\right), \\
\left\langle\left(\Delta q_{l x}\right)^{2 n+1}\right\rangle= & \left\langle\left(\Delta q_{l y}\right)^{2 n+1}\right\rangle=\left\langle\left(\Delta q_{l z}\right)^{2 n+1}\right\rangle=0,
\end{aligned}
$$

$\langle V\rangle$ is written in a Taylor series

$$
\langle V\rangle=\sum_{j>k}^{N}\left\langle V_{j k}\right\rangle \equiv \sum_{j>k}^{N} \sum_{n=0}^{\infty} V_{j k}^{(2 n)}
$$

where the $2 n$ th-order term $V_{j k}^{(2 n)}$ is expressed as

$$
V_{j k}^{(2 n)}=\frac{1}{n !}\left[\sum_{l=j, k} \frac{\rho_{l}^{2}}{2}\left(\frac{\partial^{2}}{\partial r_{l x}^{2}}+\frac{\partial^{2}}{\partial r_{l y}^{2}}+\frac{\partial^{2}}{\partial r_{l z}^{2}}\right)\right]^{n} V_{j k}\left(r_{j k}\right) .
$$

Since the pair function $V_{j k}$ depends only on the distance $r_{j k}$, the Laplacian in the Cartesian coordinates in Eq. (A10) can be reduced to the first and second derivatives with respect to the distance as

$$
\frac{\partial^{2}}{\partial r_{l x}^{2}}+\frac{\partial^{2}}{\partial r_{l y}^{2}}+\frac{\partial^{2}}{\partial r_{l z}^{2}}=\frac{\partial^{2}}{\partial r_{j k}^{2}}+\frac{2}{r_{j k}} \frac{\partial}{\partial r_{j k}}
$$

for $l=j$ and $k$. Finally, This leads to

$$
\begin{aligned}
& V_{j k}^{(2 n)}= \\
& \frac{1}{n !}\left(\frac{\rho_{j}^{2}+\rho_{k}^{2}}{2}\right)^{n}\left(\frac{\partial^{(2 n)}}{\partial r_{j k}^{(2 n)}}+\frac{2 n}{r_{j k}} \frac{\partial^{(2 n-1)}}{\partial r_{j k}^{(2 n-1)}}\right) V_{j k}\left(r_{j k}\right),
\end{aligned}
$$

for $n \geq 1$, and $V_{j k}^{(0)}=V_{j k}\left(r_{j k}\right)$. The zeroth-order term $V_{j k}^{(0)}$ corresponds to the classical representation of the pair potential function, whereas the higher-order terms $V_{j k}^{(2 n)}$ with $n \geq 1$ include quantum effects.

\section{Extended semiquantal potential functions}

Here we derive the potential expectation $\langle V\rangle$ by Eq. (A9) for the q-SPC/Fw model that consists of intramolecular stretching and bending potentials and intermolecular Coulomb and LJ potentials.

The classical form of $\mathrm{LJ}$ function is

$$
V_{\mathrm{LJ}}^{(0)}=\sum_{j>k} 4 \epsilon_{\mathrm{OO}}\left[\left(\frac{\sigma_{\mathrm{OO}}}{R_{j k}}\right)^{12}-\left(\frac{\sigma_{\mathrm{OO}}}{R_{j k}}\right)^{6}\right],
$$

where $R_{j k}$ is the distance between the $j$ th and $k$ th oxygen atoms. $\epsilon_{\mathrm{OO}}=0.1554 \mathrm{kcal} \mathrm{mol}^{-1}$ and $\sigma_{\mathrm{OO}}=3.1655 \AA$ are the LJ parameters. The corresponding second- and fourthorder SQ potential functions are derived as

$V_{\mathrm{LJ}}^{(2)}=\sum_{j>k} 12 \epsilon_{\mathrm{OO}}\left[22\left(\frac{\sigma_{\mathrm{OO}}}{R_{j k}}\right)^{12}-5\left(\frac{\sigma_{\mathrm{OO}}}{R_{j k}}\right)^{6}\right] \frac{\rho_{\mathrm{O}_{j}}^{2}+\rho_{\mathrm{O}_{k}}^{2}}{R_{j k}^{2}}$

and

$$
\begin{gathered}
V_{\mathrm{LJ}}^{(4)}= \\
\sum_{j>k} 84 \epsilon_{\mathrm{OO}}\left[143\left(\frac{\sigma_{\mathrm{OO}}}{R_{j k}}\right)^{12}-10\left(\frac{\sigma_{\mathrm{OO}}}{R_{j k}}\right)^{6}\right]\left(\frac{\rho_{\mathrm{O}_{j}}^{2}+\rho_{\mathrm{O}_{k}}^{2}}{R_{j k}^{2}}\right)^{2},
\end{gathered}
$$

where $\rho_{\mathrm{O}_{j}}$ denotes the WP width of the $j$ th oxygen atom.

In the case of the Coulomb interaction, the $2 n$ th-order coefficients for $n \geq 1$ in the Taylor series vanish from Eq. (A12), and thus we cannot apply the Taylor expansion. Instead, we can obtain the full-order SQ potential for the Coulomb interaction by performing the Gaussian integral directly, which has been derived previously ${ }^{28,49}$. To calculate the long-range electrostatic interactions without ordinary truncation, we newly derive the SQ potential with the Ewald summation method whose classical form is

$$
\begin{gathered}
V_{\text {Ewald }}^{(0)}=V_{\mathrm{el} 1}^{(0)}+V_{\mathrm{el} 2}^{(0)}+V_{\mathrm{el} 3}^{(0)}, \\
V_{\mathrm{el} 1}^{(0)}=\sum_{j>k} \sum_{a, b} \frac{1}{4 \pi \epsilon_{0}} \frac{Q_{j a} Q_{k b}}{r_{j a k b}} \operatorname{erfc}\left(\alpha r_{j a k b}\right), \\
V_{\mathrm{el} 2}^{(0)}=\frac{1}{4 \pi \epsilon_{0}} \frac{2 \pi}{L^{3}} \sum_{\mathbf{G} \neq \mathbf{0}} \frac{1}{|\mathbf{G}|^{2}} \exp \left(-\frac{|\mathbf{G}|^{2}}{4 \alpha^{2}}\right) \\
\times\left|\sum_{j} \sum_{a} Q_{j a} \exp \left(i \mathbf{G} \cdot \mathbf{r}_{j a}\right)\right|^{2}, \\
V_{\mathrm{el} 3}^{(0)}=-\frac{1}{4 \pi \epsilon_{0}} \sum_{j}\left[\frac{\alpha}{\sqrt{\pi}} \sum_{a} Q_{j a}^{2}\right. \\
\left.+\sum_{a>b} \frac{Q_{j a} Q_{j b}}{r_{j a j b}} \operatorname{erf}\left(\alpha r_{j a j b}\right)\right],
\end{gathered}
$$


where $r_{j a k b}$ is the distance between point charges $Q_{j a}$ and $Q_{k b}$, the $a$ th and $b$ th charges on the $j$ th and $k$ th molecules. Charges of $+0.42 e$ and $-0.84 e$ are placed on the hydrogen and oxygen atoms of each molecule. $L, \alpha$, and $\mathbf{G}$ are the length of the cubic simulation box, the screening parameter in the Ewald summation, and the reciprocal lattice vector, respectively. The corresponding full-order SQ representations are derived as

$$
\begin{aligned}
V_{\mathrm{el} 1}^{(\mathrm{full})}= & \sum_{j>k} \sum_{a, b} \frac{1}{4 \pi \epsilon_{0}} \frac{Q_{j a} Q_{k b}}{r_{j a k b}}\left[\operatorname{erf}\left\{\frac{r_{j a k b}}{\sqrt{2\left(\rho_{j a}^{2}+\rho_{k b}^{2}\right)}}\right\}\right], \\
& \left.-\operatorname{erf}\left\{\frac{\alpha r_{j a k b}}{\sqrt{1+2 \alpha^{2}\left(\rho_{j a}^{2}+\rho_{k b}^{2}\right)}}\right\}\right] \\
V_{\mathrm{el} 2}^{(\mathrm{full})}= & \frac{1}{4 \pi \epsilon_{0}} \frac{2 \pi}{L^{3}} \sum_{\mathbf{G} \neq \mathbf{0}} \frac{1}{|\mathbf{G}|^{2}} \exp \left(-\frac{|\mathbf{G}|^{2}}{4 \alpha^{2}}\right) \\
& \times\left[\left|\sum_{j} \sum_{a} Q_{j a} \exp \left(-\frac{|\mathbf{G}|^{2} \rho_{j a}^{2}}{2}+i \mathbf{G} \cdot \mathbf{r}_{j a}\right)\right|^{2}\right. \\
& \left.+\sum_{j} \sum_{a} Q_{j a}^{2}\left\{1-\exp \left(-|\mathbf{G}|^{2} \rho_{j a}^{2}\right)\right\}\right], \\
V_{\mathrm{el} 3}^{(\text {full })}= & -\frac{1}{4 \pi \epsilon_{0}} \sum_{j}\left[\frac{\alpha}{\sqrt{\pi}} \sum_{a} Q_{j a}^{2}\right. \\
& \left.+\sum_{a>b} \frac{Q_{j a} Q_{j b}}{r_{j a j b}} \operatorname{erf}\left\{\frac{\alpha r_{j a j b}}{\sqrt{1+2 \alpha^{2}\left(\rho_{j a}^{2}+\rho_{j b}^{2}\right)}}\right\}\right]
\end{aligned}
$$

The intramolecular potential of the $\mathrm{q}-\mathrm{SPC} / \mathrm{Fw}$ model is described by the form

$$
V_{\text {intra }}=\sum_{j}\left[V_{\text {stretch }}\left(r_{\mathrm{OH}_{1}}\right)+V_{\text {stretch }}\left(r_{\mathrm{OH}_{2}}\right)+V_{\text {bend }}\left(\theta_{\mathrm{HOH}}\right)\right],
$$

where $V_{\text {stretch }}$ and $V_{\text {bend }}$ denote the stretching and bending functions of the $j$ th molecule. Both have the harmonic form as described later. $r_{\mathrm{OH}}$ and $\theta_{\mathrm{HOH}}$ are the $\mathrm{O}-\mathrm{H}$ distance and $\mathrm{H}-\mathrm{O}-$ $\mathrm{H}$ angle in the $j$ th molecule. Note that we omit the subscript $j$ for simplicity.

The classical form of the stretching potential function is

$$
V_{\text {stretch }}^{(0)}=\frac{k_{\mathrm{s}}}{2}\left(r_{\mathrm{OH}}-r_{\mathrm{OH}}^{(\mathrm{eq})}\right)^{2}
$$

where $k_{\mathrm{s}}=1059.162 \mathrm{kcal} \mathrm{mol}^{-1} \AA^{-2}$ and $r_{\mathrm{OH}}^{(\mathrm{eq})}=1.0 \AA$. From Eq. (A12), we find the corresponding second-order quantal term is

$$
V_{\text {stretch }}^{(2)}=\frac{k_{\mathrm{s}}}{2}\left(3-\frac{2 r_{\mathrm{OH}}^{(\mathrm{eq})}}{r_{\mathrm{OH}}}\right)\left(\rho_{\mathrm{O}}^{2}+\rho_{\mathrm{H}}^{2}\right),
$$

and the higher-order terms $V_{\text {stretch }}^{(2 n)}$ for $n \geq 2$ vanish. Therefore, the second-order expansion is the exact full-order in the harmonic case.

The classical form of the bending potential function is

$$
V_{\mathrm{bend}}^{(0)}=\frac{k_{\mathrm{b}}}{2}\left(\theta_{\mathrm{HOH}}-\theta_{\mathrm{HOH}}^{(\mathrm{eq})}\right)^{2} \equiv \frac{k_{\mathrm{b}}}{2} \Delta \theta_{\mathrm{HOH}}^{2}
$$

where $k_{\mathrm{b}}=75.90 \mathrm{kcal} \mathrm{mol}^{-1} \mathrm{rad}^{-2}$ and $\theta_{\mathrm{HOH}}^{(\mathrm{eq})}=112.0^{\circ}$. In the present study, we employ the second-order approximation that yields

$$
\begin{aligned}
V_{\text {bend }}^{(2)}= & \frac{k_{\mathrm{b}}}{2}\left(1+\Delta \theta_{\mathrm{HOH}} \cdot \cot \theta_{\mathrm{HOH}}\right) \\
& \times\left(\frac{\rho_{\mathrm{O}}^{2}+\rho_{\mathrm{H}_{1}}^{2}}{r_{\mathrm{OH}_{1}}^{2}}+\frac{\rho_{\mathrm{O}}^{2}+\rho_{\mathrm{H}_{2}}^{2}}{r_{\mathrm{OH}_{2}}^{2}}-\frac{2 \rho_{\mathrm{O}}^{2}}{\left(\mathbf{r}_{\mathrm{OH}_{1}} \cdot \mathbf{r}_{\mathrm{OH}_{2}}\right)}\right) \\
& +k_{\mathrm{b}} \sin ^{2} \theta_{\mathrm{HOH}} \frac{\rho_{\mathrm{O}}^{2}}{\left(\mathbf{r}_{\mathrm{OH}_{1}} \cdot \mathbf{r}_{\mathrm{OH}_{2}}\right)} .
\end{aligned}
$$

Note that if we neglect the WP width variables of oxygen atoms $\rho_{\mathrm{O}}$, the second-order SQ potential functions in Eqs. (A25) and (A27) become equivalent to those previously reported (see Eqs. (22) and (23) of Ref. ${ }^{28}$, respectively) ${ }^{50}$.

\section{Radial distribution functions}

Here we derive the quantum RDF in a single-component system for simplicity. Note that generalization to a multicomponent system is straightforward. If the system is homogeneous and isotropic, the spatial correlation between two particles is described by the quantum mechanical expectation value of RDF,

$$
\begin{aligned}
g(r) & =\frac{1}{4 \pi d N r^{2}} \sum_{j \neq k}\left\langle\delta\left(r-\left|\mathbf{q}_{j}-\mathbf{q}_{k}\right|\right)\right\rangle \\
& =\frac{1}{4 \pi d N r} \sum_{j \neq k} \frac{1}{r_{j k} \sqrt{2 \pi\left(\rho_{j}^{2}+\rho_{k}^{2}\right)}} \exp \left[-\frac{\left(r-r_{j k}\right)^{2}}{2\left(\rho_{j}^{2}+\rho_{k}^{2}\right)}\right],
\end{aligned}
$$

where $d$ is the number density of the system.

\footnotetext{
${ }^{1}$ Bagchi, B. Chem Rev 2005, 105, 3197.

${ }^{2}$ Ball, P. Chem Rev 2008, 108, 74.
}

${ }^{3}$ Rey, R.; Møller, K. B.; Hynes, J. T. Chem Rev 2004, 104, 1915.

${ }^{4}$ Kraemer, D.; Cowan, M. L.; Paarmann, A.; Huse, N.; Nibbering, 
E. T. J.; Elsaesser, T.; Miller, R. J. D. Proc Nat Acad Sci USA 2008, 105, 437.

${ }^{5}$ Roberts, S. T.; Ramasesha, K.; Tokmakoff, A. Acc Chem Res 2009, 42, 1239

${ }^{6}$ Bakker, H. J.; Skinner, J. L. Chem Rev 2010, 110, 1498.

7 Ohmine, I.; Tanaka, H. Chem Rev 1993, 93, 2545.

8 Ando, K.; Kato, S. J Chem Phys 1991, 95, 5966.

9 Ando, K. J Chem Phys 2001, 114, 9470.

${ }^{10}$ Poulsen, J. A.; Nyman, G.; Rossky, P. J. Proc Nat Acad Sci USA $2005,102,6709$

${ }_{11}$ Morrone, J. A.; Car, R. Phys Rev Lett 2008, 101, 017801.

12 Vega, C.; Conde, M. M.; McBride, C.; Abascal, J. L. F.; Noya, E. G.; Ramirez, R.; Sesé, L. M. J Chem Phys 2010, 132, 046101.

13 Paesani, F; Yoo, S; Bakker, H. J.; Xantheas, S. S. J Phys Chem Lett 2010, 1, 2316.

14 Borysow, J.; Moraldi, M.; Frommhold, L. Mol Phys 1985, 56, 913.

15 Ando, K. J Chem Phys 1997, 107, 4585.

16 Egorov, S. A.; Everitt, K. F.; Skinner, J. L. J Phys Chem A 1999, $103,9494$.

17 Cao, J.; Voth, G. A. J Chem Phys 1994, 101, 6168.

18 Craig, I. R.; Manolopoulos, D. E. J Chem Phys 2004, 121, 3368.

${ }^{19}$ Habershon, S.; Fanourgakis, G. S.; Manolopoulos, D. E. J Chem Phys 2008, 129, 074501

${ }^{20}$ Witt, A.; Ivanov, S. D.; Shiga, M.; Forbert, H.; Marx, D. J Chem Phys 2009, 130, 194510.

21 Yoshimori, A. J Chem Phys 2008, 128, 234105.

22 Richardson, J. O.; Althorpe, S. C. J Chem Phys 2009, 131, 214106.

${ }^{23}$ Kubo, R.; Toda, M.; Hashitsume, N. Statistical Physics II: Nonequilibrium Statistical Mechanics; Springer: New York, 1985.

${ }^{24}$ Kluk, E.; Herman, M. F.; Davis, H. L. J Chem Phys 1986, 84, 326.

${ }^{25}$ Liu, J.; Miller, W. H.; Paesani, F.; Zhang, W.; Case, D. A. J Chem Phys 2009, 131, 164509.

${ }^{26}$ Meyer, H. D.; Gatti, F.; Worth, G. A., Eds.; Multidimensional Quantum Dynamics: MCTDH Theory and Applications; WileyVCH: Weilheim, 2009.

27 Wang, H.; Thoss, M. J Chem Phys 2003, 119, 1289.

${ }^{28}$ Hyeon-Deuk, K.; Ando, K. J Chem Phys 2009, 131, 064501.

${ }^{29}$ Hyeon-Deuk, K.; Ando, K. J Chem Phys 2010, 132, 164507.

${ }^{30}$ Heller, E. J. J Chem Phys 1975, 62, 1544.

31 Ando, K. J Chem Phys 2004, 121, 7136.
32 Ando, K. J Chem Phys 2006, 125, 014104.

33 Sakumichi, N.; Ando, K. J Chem Phys 2008, 128, 164516.

${ }^{34}$ Prezhdo, O. V. Theor Chem Acc 2006, 116, 206.

35 Shigeta, Y. Bull Chem Soc Jpn 2009, 82, 1323.

${ }^{36}$ Laage, D.; Hynes, J. T. Science 2006, 311, 832.

37 Ji, M.; Odelius, M.; Gaffney, K. J. Science 2010, 328, 1003.

38 Sasai, M.; Ohmine, I.; Ramaswamy, R. J Chem Phys 1992, 96, 3045.

39 Kuharski, R. A.; Rossky, P. J. J Chem Phys 1985, 82, 5164.

${ }^{40}$ Hart, R. T.; Benmore, C. J.; Neuefeind, J.; Kohara, S.; Tomberli, B.; Egelstaff, P. A. Phys Rev Lett 2005, 94, 047801.

${ }^{41}$ Paesani, F.; Zhang, W.; Case, D. A.; Cheatham III, T. E. C.; Voth, G. A. J Chem Phys 2006, 125, 184507.

${ }^{42}$ Luzar, A.; Chandler, D. Phys Rev Lett 1996, 76, 928.

${ }^{43}$ Habershon, S.; Markland, T. E.; Manolopoulos, D. E. J Chem Phys 2009, 131, 024501.

${ }^{44}$ Krynick, K.; Green, C. D.; Sawyer, D. W. Farad Discuss Chem Soc 1978, 66, 199.

45 Milotti, E. 1/f noise: a pedagogical review, http://arxiv.org/abs/physics/0204033, 2002.

46 Mudi, A.; Chakravarty, C.; Ramaswamy, R. J Chem Phys 2005, $122,104507$.

${ }^{47}$ Netz, P. A.; Starr, F. W.; Barbosa, M. C.; Stanley, H. E. Physica A $2002,314,470$

${ }^{48}$ To be accurate, $\sqrt{3} \rho_{j}$ and $\sqrt{3} \Pi_{j}$ are canonically conjugate variables in the extended phase space described by the Hamiltonian in Eq. (A4). In contrast to a one-dimensional system, the additional factor $\sqrt{3}$ appears in the WP width variables due to the spherically symmetric constrains.

${ }^{49}$ Referring to Eq. (24) of Ref. ${ }^{28}$, the nuclear quantum effect arising from the charge-charge electrostatic interaction is described by the value of the error function (erf) term that depends on the ratio of the interparticle distance to the WP widths; the smaller the ratio, the more significant the quantum effect. However, we found that the WP widths are smaller than the intermolecular distances by at least one order of magnitude in liquid water at ambient conditions, and as a result the erf term becomes the saturated value of unity (i.e., classical limit); in fact, the deviation $(1-$ erf) is found to be always smaller than $10^{-14}$.

${ }^{50}$ Note that typos exist in Eqs. (22) and (23) of Ref. ${ }^{28}$; that is, $1 / 2$ was missing in the right terms of these equations although actual calculations were performed based on the correct equations. 